\section{Intraocular lens exchange for high myopia in pseudophakic children}

\begin{abstract}
Purpose The purpose of this study was to examine the preoperative factors and postoperative outcomes following intraocular lens (IOL) exchange for high myopia in pseudophakic children.

Methods The medical records of all patients undergoing IOL exchange for high myopia were retrospectively reviewed.

Results A total of $\mathbf{1 5}$ eyes were identified that had undergone an IOL exchange for myopic shift. Average age of cataract extraction (CE) was 5.4 months. In all, 10/15 had a unilateral cataract. IOL exchange usually occurred at an average of 6 years following cataract surgery. The average spherical equivalent (SE) of the refractive error at that time was $-9.6 \mathrm{D}$. Following IOL exchange, SE was -1.3 D. A two-line reduction in best-corrected visual acuity was observed in $1 / 13$ of our patients for whom pre- and post-exchange data were available. The average axial length $(A L)$ of the eye undergoing the IOL exchange was $24.0 \mathrm{~mm}$, average $\mathrm{AL}$ in the non-operative eye was $22.1 \mathrm{~mm}$. On average, the operative eyes grew $4.4 \mathrm{~mm}$ and the non-operative eyes $3.02 \mathrm{~mm}$. No adverse events were seen in the operative eyes.

Conclusion Younger age at the time of CE creates a greater likelihood of AL elongation and predisposes a child to myopic shift. IOL exchange should be considered an option to reduce anisometropia and associated aniseikonia to improve visual outcomes. Successful visual rehabilitation and predictable post-exchange refractions were seen with our patients.

Eye (2016) 30, 1199-1203; doi:10.1038/eye.2016.152; published online 15 July 2016
\end{abstract}

\section{Introduction}

Today's microsurgical devices and instruments have led to consistent advancement in pediatric cataract surgery. It has become a safe and reliable operation for even the youngest patient. Consequently, the focus has turned toward optimizing refractive outcomes. Spectacles, contact lenses, and intraocular lens (IOL) implantation have all been used for correction of surgically induced aphakia following pediatric cataract surgery. Primary IOL implantation is an accepted surgical strategy in children. ${ }^{1}$ It provides a stable retinal image with minimal aniseikonia and offers a permanent method of optical correction.

Understanding the growth and development of a child's eye is essential to optimize IOL selection. Gordon and Donzis, ${ }^{2}$ in their study of the normal refractive development of the human eye, found that the globe axial length (AL) increases markedly in the first few years after birth and is within $1.0 \mathrm{~mm}$ of adult length by 5 to 6 years of age. A rapid postnatal growth phase of $3.8 \mathrm{~mm}$ in the first year of life, a slower infantile growth phase of $1.2 \mathrm{~mm}$ up to 5 years of age, followed by a slow juvenile growth phase of $1.4 \mathrm{~mm}$ up to 13 years of age has been described. ${ }^{2}$ For this reason, when an IOL is placed in infancy there is a degree of undercorrection (or a deliberately hyperopic postoperative refractive aim) to account for the myopic shift that takes place with increasing AL. Varying degrees of myopic shift have been reported. ${ }^{3}$ This individual variability seems most difficult to predict in the youngest patient population. For example, some patients implanted under age of 1 year may only shift $1 \mathrm{D}$, whereas others may shift $10 \mathrm{D}$ or more. ${ }^{4}$

Ideally, the foremost goal of primary IOL implantation should be to facilitate visual development and reduce amblyopia in a developing eye. An important secondary goal is to select an IOL that will not result in significant anisometropia in unilateral cases or high myopia following the period of rapid eye
${ }^{1}$ Wilmer Eye Institute, Johns Hopkins University, Baltimore, MD, USA

${ }^{2}$ Department of Ophthalmology, Miles Center for Pediatric Ophthalmology, Storm Eye Institute, Medical University of South Carolina, Charleston, SC, USA

Correspondence: ME Wilson, Department of Ophthalmology, Miles Center for Pediatric Ophthalmology, Storm Eye Institute, Medical University of South Carolina, 167 Ashley Avenue, Charleston, SC 29425-5536, USA

Tel: +1 843792 7622:

Fax: +1 8437928289

E-mail: wilsonme@musc.edu

Received: 25 February 2016 Accepted in revised form: 21 June 2016 Published online: 15 July 2016 
growth. Understanding whether common factors exist that may predispose certain patients to a significant myopic shift will help future IOL selection. In the absence of the ability to predict postoperative refraction with near certainty, the feasibility and success of IOL exchange in highly myopic patients is important to establish.

We report the preoperative factors and postoperative outcomes of 15 eyes in children undergoing IOL exchange due to myopic shift.

\section{Materials and methods}

A retrospective chart review of all eligible patients undergoing treatment by a single surgeon (MEW) at the Storm Eye Institute, Medical University of South Carolina (MUSC) between 1992 and 2013 was performed. The institutional review board of MUSC approved the study. Medical records of patients with a primary IOL placed in childhood who had a myopic shift in refraction sufficient enough to require a subsequent operation to exchange the lens were reviewed. Myopic shift was defined as clinically significant if the spherical equivalent (SE) was $>-6.5$ without subjective complaints or $<-6.5$ with significant aniseikonia. Those patients undergoing IOL exchange or revision for other reasons (eg, IOL dislocation or decentration) were not included.

Refractive error was obtained using a variety of methods, including retinoscopy and manual refraction with subjective refinement. In addition, slit-lamp examinations were conducted at all follow-up appointments, with attention to lens position, degree of inflammation, intraocular pressure, and fundoscopic examination.
The patients were followed in the clinic on postoperative day 1 , week 1 , month 1 and then variable intervals (at the least every 6 months). In addition, AL measurements and keratometry were attempted at least once a year. If unable to be obtained in clinic, an examination under anesthesia was performed.

IOL power calculations were based on the ocular biometry measurements obtained at the time of cataract extraction. The Holladay 1 formula was used for calculations with optimization using a surgeon factor. Lens selection and targeted refraction was at the discretion of the surgeon. The difference between the postoperative refraction recorded 6 weeks following initial IOL implantation and the postoperative refraction at the last follow-up was recorded and used to calculate the amount of myopic shift. The preoperative AL was used as a baseline to determine the change in AL. The possibility of glaucoma was excluded by intraocular pressure measurement and disc evaluation during follow-ups.

\section{Results}

Twenty eyes were identified that had undergone an IOL exchange. Fifteen of the 20 were for myopic shift. The remaining 5 were for IOL dislocation and therefore, were excluded from the study. Records following the exchange were available for a period ranging from 4 months to 14 years, with an average of 3 years. Ten of the 15 had presented with unilateral cataract. The remaining 5 eyes represented 1 eye in cases of bilateral cataracts. The average age at cataract extraction (CE) was 5.5 months, range $0.9-24.5$ months. Table 1 shows the age at cataract surgery, type and location of the primary IOL and, when available, the targeted refraction. Average

Table 1 Demographics and preoperative characteristics

\begin{tabular}{|c|c|c|c|c|c|c|}
\hline ID & Age at CE (months) & Age at IOL (months) & OD/OS & Bilateral us unilateral & Primary IOL & Targeted refraction $(D)$ \\
\hline 1 & 1.8 & NA & OS & Bilateral & Rayner (bag) & +7.00 \\
\hline 2 & 1.2 & NA & OS & Unilateral & MA60 (bag) & +6.00 \\
\hline 3 & 1.2 & NA & OS & Bilateral & SN60 (bag); SA30 (sulcus) & +8.00 \\
\hline 4 & 24.5 & NA & OS & Unilateral & MC60 (sulcus) & ND \\
\hline 5 & 0.9 & NA & OS & Unilateral & SN60 (bag) & +8.50 \\
\hline 6 & 3.0 & 10 & OS & Unilateral & MA60 (bag) & -0.50 \\
\hline 7 & 7.6 & NA & OD & Unilateral & SN60 (bag) & +8.00 \\
\hline 8 & 1.9 & NA & OD & Bilateral & SN60 (bag) & +8.00 \\
\hline 9 & 3.0 & 82.9 & OS & Bilateral & U85JS (sulcus) & ND \\
\hline 10 & 15.0 & 23.8 & OD & Unilateral & MC60 (sulcus) & ND \\
\hline 11 & 6.0 & NA & OS & Unilateral & MA60 (bag) & ND \\
\hline 12 & 5.3 & NA & OD & Unilateral & $722 \mathrm{C}$ (sulcus) & -0.50 \\
\hline 13 & 3.4 & 42 & OD & Bilateral & SA60 (bag) & Emmetropia \\
\hline 14 & 2.0 & 45.6 & OS & Unilateral & MA60 (bag) & +2.00 \\
\hline 15 & ND & NA & OD & Unilateral & MA60 (ND) & ND \\
\hline
\end{tabular}

Abbreviations: CE, cataract extraction; NA, not applicable; ND, no data. ${ }^{\text {a }}$ If different than age at cataract extraction. 
post-operative targeted refraction was $+4.65 \mathrm{D}$. Where targeted refraction is not listed, these represent patients whose initial CE and IOL placement was performed elsewhere, but the information on age and IOL was available. One eye is missing from this table as CE/IOL placement was performed elsewhere, and we assumed care for IOL exchange.

IOL removal took place an average of 7.1 years following cataract surgery. Table 2 shows pre- and postoperative refractions following the IOL exchange. For those eyes identified as having high myopia necessitating IOL removal, the average SE of the refractive error was - 9.6 $\mathrm{D}$, reflecting an average change in refraction of 14.25 D. There was a slight, but non-statistically significant, trend for less myopic shift to take place in those eyes where the IOL was implanted at the time of initial cataract surgery compared with those who received an IOL later in the treatment course (-8.67 vs - 10.1, respectively). Following IOL exchange, the average SE of the refractive error was $-1.3 \mathrm{D}$. A reduction in best-corrected visual acuity was observed in 1/13 of our patients for whom pre- and post-exchange data were available (Table 3 ).

AL measurements at the time of primary surgery, if performed at our institution, and at IOL exchange were reviewed. The average AL of the eye undergoing the IOL exchange ('operative') was $24 \mathrm{~mm}$. In the other ('non-operative') eye-a phakic eye in the 10 unilateral cataracts and pseudophakic in the 5 bilateral cases-the average AL was $22.1 \mathrm{~mm}$. The amount of eye growth from the time of primary IOL placement to IOL exchange was calculated. On average, the operative eyes grew $4.4 \mathrm{~mm}$ and the non-operative eyes $3.02 \mathrm{~mm}$.

No adverse events were seen in the operative eyes following IOL exchange. Specifically, prolonged postoperative inflammation, glaucoma, visual axis opacification, infection, or unexpected refractive outcomes were not seen in a follow-up period ranging from 4 months to 14 years.

\section{Discussion}

The acceptance of IOL implantation as an acceptable method of optical correction at cataract surgery in children $<2$ years of age increased among pediatric eye surgeons from 12.9 to $81.9 \%$ from 1993 to 2001 . $^{1}$ Recent outcomes from the Infant Aphakia Treatment Study (IATS) demonstrated no difference in visual outcomes at age 5 years between those implanted with an IOL in infancy and those left aphakic and optically corrected with a contact lens. The authors concluded that selective use of IOLs in this population would be acceptable, specifically recommending them for situations where contact lens (CTL) use is an anticipated challenge. ${ }^{5}$

A risk factor for pseudophakic high myopia includes excessive axial growth and this phenomenon occurs in situations such as form vision deprivation, unilateral

Table 3 Visual acuity before and after IOL exchange

\begin{tabular}{lll}
\hline ID & Pre-exchange $V A$ & Post-exchange $V A$ \\
\hline 1 & FF & Brief fix \\
2 & $20 / 30$ & $20 / 30$ \\
3 & CUSUM & CF \\
4 & $20 / 200$ & $20 / 125$ \\
5 & FF & LP \\
6 & OD pref & HM \\
7 & $20 / 70$ & $20 / 70$ \\
8 & $20 / 500$ & CF \\
9 & $20 / 25$ & $20 / 25$ \\
10 & CSUM & $20 / 250$ \\
11 & $20 / 400$ & $20 / 100$ \\
12 & FF & $20 / 30+$ \\
15 & CF @ 3ft & $20 / 400$ \\
\hline
\end{tabular}

Table 2 Intraocular exchange: age and refraction

\begin{tabular}{lccc}
\hline ID & Age IOL Exchange $(\mathrm{ms})$ & Pre-exchange refraction $(\mathrm{SE})$ & Post-exchange refraction (SE) \\
\hline 1 & 9.1 & $-12.00+4.50 \times 80(-9.75)$ & $-2.00+1.00 \times 100(-1.5)$ \\
2 & 95 & $-8.50+0.25 \times 170(-8.5)$ & $-4.50+1.50 \times 15(-3.75)$ \\
3 & 49.3 & $-7.50+5.00 \times 80(-5.0)$ & $-4.00 \mathrm{sph}$ \\
4 & 11 & $-9.25+1.75 \times 125(-8.25)$ & $-2.50+1.00 \times 165(-2.0)$ \\
5 & 9 & $-7.50+1.00 \times 100(-7.0)$ & $+1.00+1.25 \times 100(-1.625)$ \\
6 & 41 & $-18.5 \mathrm{sph}$ & $-1.00+4.50 \times 165(+1.25)$ \\
7 & 88.8 & $-7.50+1.50 \times 90(-6.75)$ & $-1.00+0.75 \times 090(-0.625)$ \\
8 & 60.8 & $-7.50+1.50 \times 90(-6.75)$ & $-1.25+0.25 \times 89(-1.125)$ \\
9 & 183.4 & $-9.00+4.00 \times 75(-7.0)$ & $-0.75+1.25 \times 85(-0.125)$ \\
10 & 130.6 & $-10.50+2.00 \times 95(-9.5)$ & $-3.50+0.50 \times 135(-3.25)$ \\
11 & 122.5 & $-14.5 \mathrm{sph}$ & $-1.00+1.00 \times 90(-0.5)$ \\
12 & 24.1 & $-12.00+1.00 \times 90(-11.5)$ & $-6.25 \mathrm{sph}$ \\
13 & 162 & $-8.50+2.25 \times 005(-7.50)$ & $+1.00+2.00 \times 95(+2.0)$ \\
14 & 188.4 & $-10.00+4.00 \times 90(-8.00)$ & $+0.50 \mathrm{sph}$ \\
15 & 134.5 & $-15.50 \mathrm{sph}$ & $+1.00+2.00 \times 95(+2.0)$ \\
\hline
\end{tabular}


cataracts, amblyopia, early IOL implantation, and glaucoma. We excluded glaucoma from our review. One of the more interesting observations from our review was the wide range in both age of initial cataract surgery and targeted refraction resulting in high myopia. Although our average age of cataract surgery was young, there was an older subset that still developed a large myopic shift. Similarly, having a less hyperopic- (more myopic) targeted refraction should predictably lead to a greater incidence of myopic outcomes. However, we found the majority of our patients were undercorrected (deliberately left hyperopic) for their age of IOL implantation.

One of the limitations of this study is the failure to quantify the contribution of possible imprecision of biometry. The surgeon used the same lens equation, instrumentation, and ultrasonographer to arrive at lens selections in this cohort of patients as for others in his practice. Therefore, this population represents those who underwent a myopic shift despite standard operative approach.

It can be anticipated that the trend of primary IOL implantation will continue; in those cases of unilateral cataract, the IATS findings suggest an IOL would be an acceptable choice if economic, social, or physical factors imply poor CTL compliance should be anticipated. Initial emmetropia can counteract dense amblyopia; however, leaving less hyperopia creates high myopia as the child grows.

Unfortunately, these same issues may remain if the child goes on to progressive, high myopia. Notably, most myopic shift occurs in the first 3 years of life. ${ }^{6}$ Therefore, a child who was initially a poor CTL candidate or CTL-intolerant, spectacle intolerant, or suffering from anisometropic amblyopia not successfully managed with penalization will likely still be so when myopic shift occurs. IOL exchange should be considered an option to mitigate adverse refractive outcomes.

In our case series of IOL exchanges for high myopia, timing of IOL (primary and secondary), changes in AL and interocular AL difference, and refractive outcomes were examined. We reported the average timeline for these exchanges. Successful visual rehabilitation and reliable refractive outcomes were seen in the majority of our patients.

The IATS concluded that for those children $<7$ months of age with unilateral cataract, placing a primary IOL increases the likelihood the child will have at least 1 additional intraocular surgery. ${ }^{4,7}$ Understanding this should lead the surgeon to have an informed discussion, including likelihood of additional surgery, with the caregivers prior to cataract surgery. Knowing IOL exchange is a feasible solution to potential high myopia is a valuable fact to add to this dialogue, especially in high-risk eyes.

\section{Summary}

\section{What was known before}

- Glasses, contact lenses, and intraocular lenses (IOLs) are each acceptable options for the correction of surgically induced aphakia following pediatric cataract surgery. IOLs can be safely and successfully implanted in cases of childhood cataract.

- To compensate for the anticipated myopic shift that occurs with age and growth of the eye, pediatric cataract surgeons utilize a strategy of intentional undercorrection (or deliberate post-operative hyperopic refractive aim).

- Variability exists in the amount of myopic shift that occurs with age, resulting in potential high myopia or anisometropia.

What this study adds

- IOL exchange should be considered an acceptable option in those circumstances where a large myopic shift in refractive error has occurred in a pseudophakic child.

- Visual rehabilitation, including decreasing spectacle dependence and minimizing anisometropia, can be facilitated by IOL exchange.

- IOL exchanges were performed without adverse events, including glaucoma, visual axis obscuration, infection, or unexpected refractive outcomes.

\section{Conflict of interest}

The authors declare no conflict of interest.

\section{Acknowledgements}

This study was supported by an unrestricted grant to MUSC-SEI from Research to Prevent Blindness, Inc., New York, NY, USA, redundant reference to Research to Prevent Blindness.

\section{References}

1 Wilson Jr ME, Bartholomew LR, Trivedi RH. Pediatric cataract surgery and intraocular lens implantation: practice styles and preferences of the 2001 ASCRS and AAPOS memberships. J Cataract Refract Surg 2003; 29(9): 1811-1820.

2 Gordon RA, Donzis PB. Refractive development of the human eye. Arch Ophthal 1985; 103: 785-789.

3 Crouch ER, Crouch ER, Pressman SH. Prospective analysis of pediatric pseudophakia: myopic shift and postoperative outcomes. J AAPOS 2002; 6(5): 277-282.

4 Superstein R, Archer SM, Del Monte MA. Minimal myopic shift in pseudophakic versus aphakic pediatric cataract patients. J AAPOS 2002; 6: 271-276.

5 The Infant Aphakia Treatment Study Group. Comparison of contact lens and intraocular lens correction of monocular aphakia during infancy: a randomized clinical trial of HOTV 
optotype acuity at age 4.5 years and clinical findings at age 5 years. JAMA Ophthalmol 2014; 132(6): 676-682.

6 O'Keefe M, Fenton S, Lanigan B. Visual outcomes and complications of posterior chamber intraocular lens implantation in the first year of life. J Cataract Refract Surg 2001; 27: 2006-2011.
7 Plager DA, Lynn MJ, Buckley EG, Wilson ME, Lambert SR. Infant Aphakia Treatment Study Group. Complications, adverse events, and additional intraocular surgery 1 year after cataract surgery in the Infant Aphakia Treatment Study. Ophthalmology 2011; 118: 2330-2334. 